\title{
Accretion phenomena in nearby star-forming dwarf galaxies
}

\author{
F. Annibali ${ }^{1,2}$, M. Tosi $^{1}$, A. Aloisi ${ }^{3}$, M. Bellazzini ${ }^{1}$, A. Buzzoni ${ }^{1}$, \\ M. Cignoni ${ }^{3,4}$, L. Ciotti ${ }^{2}$, F. Cusano ${ }^{1}$, C. Nipoti ${ }^{2}$, E. Sacchi ${ }^{1,2}$, \\ D. Paris ${ }^{5}$ and D. Romano ${ }^{1}$ \\ ${ }^{1}$ INAF-Osservatorio Astronomico di Bologna \\ Via Ranzani 1, I-40127 Bologna, Italy \\ email: francesca.annibali@oabo.inaf.it \\ ${ }^{2}$ Dipartimento di Fisica e Astronomia, Università di Bologna \\ viale Berti-Pichat 6/2, 40127 Bologna, Italy \\ ${ }^{3}$ Space Telescope Science Institute \\ 3700 San Martin Drive, Baltimore, MD 21218, USA \\ ${ }^{4}$ Dipartimento di Fisica, Università di Pisa \\ Largo Bruno Pontecorvo, 3, 56127 Pisa, Italy \\ ${ }^{5}$ INAF-Osservatorio Astronomico di Roma \\ Via Frascati 33, 00078, Monte Porzio Catone, Italy
}

\begin{abstract}
We present two pilot studies for the search and characterization of accretion events in star-forming dwarf galaxies. Our strategy consists of two complementary approaches: i) the direct search for stellar substructures around dwarf galaxies through deep wide-field imaging, and ii) the characterization of the chemical properties in these systems up to large galactocentric distances. We show our results for two star-forming dwarf galaxies, the starburst irregular NGC 4449, and the extremely metal-poor dwarf DDO 68.
\end{abstract}

Keywords. galaxies: individual (NGC 4449, DDO 68), galaxies: dwarf, galaxies: interactions

\section{Introduction}

Studies of accretion events in star-forming dwarf galaxies are crucial to our understanding of cosmology and galaxy evolution. Although $\Lambda \mathrm{CDM}$ models predict that substructures persist down to the resolution limit of the simulations, observational evidence of accretion events into LMC-size dwarf galaxies and those less massive than $\sim 10^{9} M_{\odot}$ in stars have so far been rare (e.g., Martínez-Delgado et al. 2012, Amorisco et al. 2014). In this context, direct observational evidence of dwarfs accreting smaller dwarfs would be important to test the self-similarity of the hierarchical formation process down to the smallest scales. Furthermore, there is a potential connection between starbursts in dwarf galaxies and accretion events; indeed, recent studies in H I (e.g., Lelli et al. 2014) have shown that disturbed H I kinematics, H I companions, and filamentary H I structures are more common in starburst dwarfs than in typical star-forming irregulars.

\section{Strategy}

We are pursuing two different, complementary approaches to characterize the occurrence of accretion events in dwarf galaxies: i) the direct detection of stellar substructures around these systems through deep wide-field imaging, and ii) the indirect detection of 

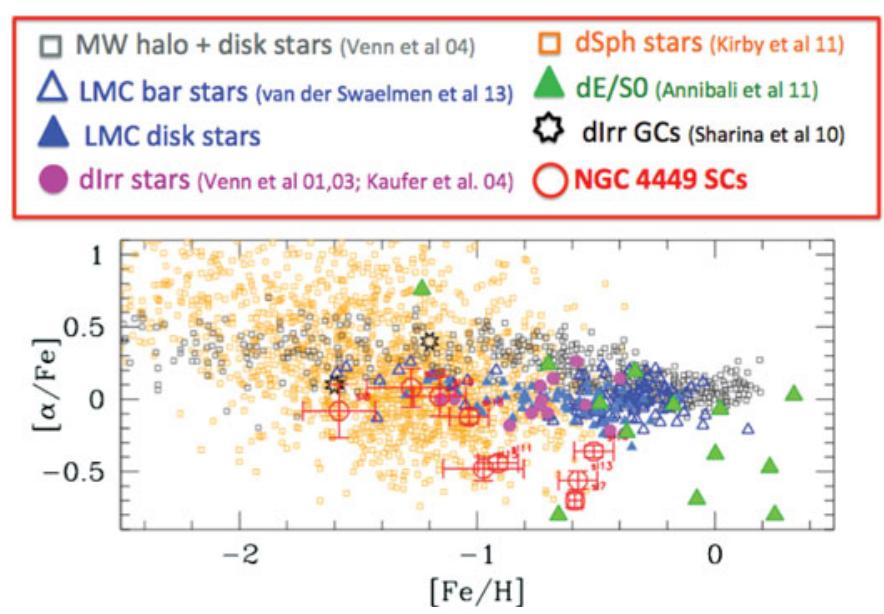

Figure 1. $[\alpha / \mathrm{Fe}]$ vs. $[\mathrm{Fe} / \mathrm{H}]$ for SCS in NGC 4449 (open circles). Compared are literature abundances for MW halo and disk stars (Venn et al. 2004), LMC bar and disk stars (Van der Swaelmen et al. 2013), dIrr stars (Venn et al. 2001, 2003; Kaufer et al. 2004), dIrr globular clusters (Sharina et al. 2010), dSph stars (Kirby et al. 2011), and dwarf early-type galaxies (Annibali et al. 2011b).

accretion events through characterization of the chemical properties up to large galactocentric distances. Both approaches require powerful 8-10 m telescopes capable of deep wide-field imaging and spectroscopy. Here we present two pilot studies demonstrating the high potential of the Large Binocular Telescope (LBT), which consists of two $\sim 8 \mathrm{~m}$ mirrors, in accomplishing our goals.

\section{Chemical properties of NGC 4449}

$\mathrm{H}$ II regions, planetary nebulae (PNe) and unresolved star clusters (SCs) in the Magellanic irregular NGC 4449 were observed on January and April, 2013 with the MultiObject Double CCD Spectrographs/Imagers (MODS) mounted on the LBT. Chemical abundances of $\mathrm{H}$ II regions and PNe were derived from nebular emission lines. SC ages, metallicities, and $[\alpha / \mathrm{Fe}]$ ratios were instead derived by comparing spectral absorption features with populations synthesis models. Fig. 1 shows the $[\alpha / \mathrm{Fe}]$ versus $[\mathrm{Fe} / \mathrm{H}]$ diagram for the SCs in NGC 4449, in comparison with literature abundances derived for stars in the Milky Way, in the Large Magellanic Cloud, in dwarf Irregulars (dIrr), dwarf Spheroidals (dSph), and in a sample of dwarf elliptical and S0 galaxies. Some of the SCs in NGC 4449 exhibit very low $[\alpha / \mathrm{Fe}]$ ratios, indicating an extremely slow and inefficient star formation; their $[\alpha / \mathrm{Fe}]$ ratios are lower than in the LMC (with a mass and structure similar to NGC 4449), and are comparable to abundance ratios derived in some dSph stars, or in the integrated spectra of dwarf E/S0 galaxies. Two of the SCs with low $[\alpha / \mathrm{Fe}]$ (namely SCs 77 and 79 in Annibali et al. 2011a) are very massive $\left(\sim 2 \times 10^{6} \mathrm{M}_{\odot}\right)$ and elliptical $(\epsilon \sim 0.2-0.3)$; SC 77 is also associated with a symmetric structure of blue stars reminiscent of tidal tails, and has already been suggested to be the nucleus of a dwarf galaxy disrupted by NGC 4449 (Annibali et al. 2012). The new chemical abundance results reinforce the hypothesis that some of the SCs in NGC 4449 are in fact the nuclei of accreted dwarf satellites. 


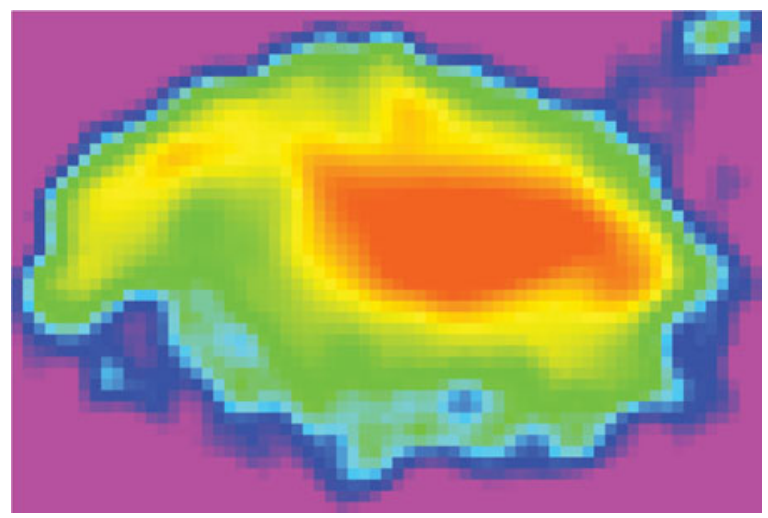

Figure 2. Spatial density map (arbitrary scale) of the stars resolved with ACS@HST in DDO 68. The FoV is $\sim 210^{\prime \prime} \times 160^{\prime \prime}$, or $\sim 13 \times 10 \mathrm{kpc}^{2}$ at a distance of $\sim 12.65 \mathrm{Mpc}$ (Sacchi et al. 2016).

\section{A newly detected stellar stream around DDO 68}

The very metal-poor $(12+\log (\mathrm{O} / \mathrm{H})=7.15$, Izotov \& Thuan 2009) star-forming dwarf DDO 68 exhibits a highly distorted morphology and a strong deviation from the massmetallicity relation, and has already been suggested to be an interacting system (Cannon et al. 2014, Tikhonov et al. 2014). We first observed DDO 68 with the ACS@HST (PI Aloisi), and later, in January 2016 , with the wide field $(\sim 23$ ' $x$ 23') Large Binocular Cameras (LBC) at LBT (PI Annibali). Fig. 2 shows the density map of the stars resolved with ACS, where the highly distorted morphology of DDO 68 is well visible. The new LBT observations revealed the presence of a stream associated with DDO 68 (Annibali et al. in prep); a portion of the stream falls within our ACS images and is resolved into individual stars (see the feature in the top right corner of Fig.2). From our star formation history study based on the HST data, the total stellar mass in DDO 68 is about $10^{8} M_{\odot}$. This makes DDO 68 the smallest dwarf galaxy with observational evidence of accretion.

\section{References}

Annibali, F., Tosi, M., Aloisi, A., \& van der Marel, R. P. 2011a AJ, 142, 129

Annibali, F., Grützbauch, R., Rampazzo, R., et al. 2011b, A\&AA, 528, A19

Annibali, F., Tosi, M., Aloisi, A., et al. 2012, ApJL, 745, L1

Amorisco, N. C., Evans, N. W., \& van de Ven, G. 2014, Nature, 507, 335

Cannon, J. M., Johnson, M., McQuinn, K. B. W., et al. 2014, ApJL, 787, L1

Izotov, Y. I., \& Thuan, T. X. 2009 ApJ, 690, 1797

Kaufer, A., Venn, K. A., Tolstoy, E., Pinte, C., \& Kudritzki, R.-P. 2004, AJ, 127, 2723

Kirby, E. N., Lanfranchi, G. A., Simon, J. D., et al. 2011, ApJ, 727, 78

Lelli, F., Verheijen, M., \& Fraternali, F. 2014, MNRAS, 445, 1694

Martínez-Delgado, D., Romanowsky, A. J., Gabany, R. J., et al. 2012, ApJL, 748, L24

Sacchi, E., Annibali, F., Cignoni, M., et al. 2016 arXiv:1604.06239

Sharina, M. E., Chandar, R., Puzia, T. H., et al. 2010, MNRAS, 405, 839

Tikhonov, N. A., Galazutdinova, O. A., \& Lebedev, V. S. 2014, Astronomy Letters, 40, 1

Van der Swaelmen, M., Hill, V., Primas, F., \& Cole, A. A. 2013, A\& A, 560, A44

Venn, K. A., Lennon, D. J., Kaufer, A., et al. 2001, ApJ, 547, 765

Venn, K. A., Tolstoy, E., Kaufer, A., et al. 2003, AJ, 126, 1326

Venn, K. A., Irwin, M., Shetrone, M. D., et al. 2004, AJ, 128, 1177 